\title{
Interferon regulatory factor 7 regulates airway epithelial cell responses to human rhinovirus infection
}

\author{
Anthony Bosco ${ }^{1 * \dagger}$, Shahina Wiehler ${ }^{2 \dagger}$ and David Proud ${ }^{2}$
}

\begin{abstract}
Background: Human rhinoviruses (HRV) cause the majority of colds and trigger exacerbations of chronic lower airway diseases. Airway epithelial cells are the primary site for HRV infection and replication, and the initiation of host inflammatory responses. At present, the molecular mechanisms that underpin HRV responses in airway epithelial cells are incompletely understood. The aim of this study was to employ microarray profiling, upstream regulator analysis, and siRNA mediated gene silencing to further our understanding of the role of interferon regulatory factor 7 (IRF7) in this response.
\end{abstract}

Methods: Primary human bronchial epithelial cells (HBE) where transfected with siRNA that targets IRF7 or a nonsilencing control (all-star control) using Lipofectamine. The cells were allowed to recover, and then cultured in the presence or absence of HRV-16 for $24 \mathrm{~h}$. Global patterns of gene expression were profiled on microarrays. A subset of genes identified in the microarray study were validated at the mRNA and/or protein level using real time RT-qPCR, ELISA, and western blots.

Results: Hundreds of genes were upregulated in HBE during HRV infection. Pathways analysis demonstrated that these genes were mainly involved in type I and II interferon signaling, RIG-I/MDA5 signaling, antigen processing and presentation, and apoptosis. Upstream regulator analysis of these data suggested that IRF7 was a major molecular driver of this response. Knockdown of IRF7 reduced the HRV-driven upregulation of genes involved in antiviral responses (interferon signaling, Toll-like receptor signaling, NOD-like receptor signaling, RIG-I/MDA5 signaling), and increased the expression of genes that promote inflammation (e.g. CXCL5, IL-33, IL1RL1) and the response to oxidative stress. However, the majority of genes that were perturbed by HRV in HBE cells including those that are known to be regulated by IRF7 were insensitive to IRF7 knockdown. Upstream regulator analysis of the part of the response that was insensitive to IRF7 knockdown suggested it was driven by NF-KB, STAT1, STAT3, and IRF1.

Conclusions: Our findings demonstrate that IRF7 regulates the expression of genes involved in antiviral immunity, inflammation, and the response to oxidative stress during HRV infections in HBE cells, and also suggests that other transcription factors play a major role in this response.

Keywords: Rhinovirus, Airway epithelial cells, Interferon regulatory factor 7, Innate immunity, Gene expression profiling, Gene silencing

\footnotetext{
* Correspondence: Anthony.Bosco@telethonkids.org.au

${ }^{\dagger}$ Equal contributors

${ }^{1}$ Telethon Kids Institute, University of Western Australia, PO Box 855, West

Perth, WA 6872, Australia

Full list of author information is available at the end of the article
} 


\section{Background}

Human rhinovirus is a positive sense, single-strand RNA virus from the Picornavirus family. It causes the majority of colds, and it also triggers exacerbations of asthma, chronic obstructive pulmonary disease, and cystic fibrosis [1]. More than 150 strains of HRV have been identified, and these have been classified into three species (A, $B, C)$ based on genome sequencing data [2]. Airway epithelial cells are the primary site for rhinovirus infection and replication. Depending on the serotype of HRV, cell entry is mediated by binding to the receptors ICAM-1 (major group), low-density-lipoprotein receptor family members (minor group), or cadherin-related family member 3 (CDHR3 for HRV-C) [3].

Host responses to HRV are thought to be initiated when viral proteins and nucleic acids trigger pathogen recognition receptors (PRR) of the innate immune system (TLR2, TLR3, TLR7, TLR8, MDA5, RIG-I) [4-6]. PRR signaling triggers intracellular signaling cascades that converge on the IRF and NF- $\mathrm{kB}$ family of transcription factors, which in turn activate interferon-induced antiviral and proinflammatory gene expression programs respectively. In epithelial cells, interferon programs are mainly activated via TLR3, MDA5, and RIG-I signaling [4-6]. IRF7 plays a central role in the activation of innate antiviral responses by controlling the transcription of type I and III interferon genes [7]. Type I interferon signaling in turn upregulates IRF7 expression, thereby providing positive feedback amplification of the antiviral response [8]. The interferon system plays a crucial role in antiviral defence by upregulating a set of effector molecules (e.g. Mx1, OAS, PKR, ISG15), which induce a robust antiviral state in infected and neighbouring cells preventing the spread of infection [9]. The importance of IRF7 in antiviral immunity was clearly demonstrated in IRF7 deficient mice, which are highly susceptible to viral infections [10]. Recent data in humans indicates that null mutations in IRF7 are associated with susceptibility to severe, life-threatening influenza [11].

Although it is well established that IRF7 is a master regulator of the antiviral response, the function (or redundancy thereof) of IRF7 can vary markedly depending on the cell type and the virus [12]. We reported that expression of IRF7 was upregulated in nasal epithelial scrapings from human adults after experimental infection with HRV-16 [13]. Employing a computational analysis of gene expression profiling data derived from nasal wash samples, we also showed that IRF7 was a major hub, connecting interferon-related gene networks during picornavirus-induced asthma exacerbations in children [14]. Lewis et al. reported that expression of IRF7 was upregulated in nasal aspirates from asthmatic children during natural colds [15]. Notably, all of the above studies focused on expression levels of IRF7; this is the first study to perform gene silencing experiments in HBE to elucidate the role of IRF7 in the regulation of HRV responses.

\section{Methods \\ Materials}

The following reagents were purchased from the indicated suppliers: Ham's F-12 medium, Eagle's minimal essential medium, Hank's balanced salt solution (HBSS), penicillin-streptomycin-amphotericin B, L-glutamine, TRIzol reagent, sodium pyruvate, nonessential amino acids, gentamicin, fetal bovine serum (FBS), dNTPs, oligo $(\mathrm{dT})$, random hexamers, and Superscript III from Invitrogen Life Technologies (Burlington, Ontario, Canada). Bronchial epithelial cell growth medium (BEGM) was from BioWhittaker (Walkersville, MD). Gene expression kits for GAPDH, IL-6, CXCL5, and HERC5 were from Applied Biosystems (Foster City, CA); antibody pairs for CCL5, CXCL5, and CXCL10, as well as recombinant standards for each assay were from R\&D Systems (Minneapolis, MN); TaqMan master mix and complete protease inhibitor cocktail tablets were from Roche Diagnostics (Laval, Quebec, Canada); western blot antibody for IRF7 was from BioLegend (San Diego, CA), while the viperin antibody was from Abcam (Toronto, $\mathrm{ON}$ ). All other chemicals were purchased from SigmaAldrich (St. Louis, MO).

\section{Primary epithelial cell culture}

Normal human lungs not used for transplantation were obtained from a tissue retrieval service (International Institute for the Advancement of Medicine, Edison, NJ). Primary HBE cells from a total of 9 donors (4 male; age range 13-63 years) were used for the current studies. All donors died from head trauma or stroke, and none of the donors had any inflammatory lung disease. Written informed consent was obtained from responsible next of kin for tissues to be used for either transplant or for research. Approval to use recovered organs for these studies was obtained from the Conjoint Health Research Ethics Board of the University of Calgary. Primary HBE cells were obtained by protease digestion of dissected airways as previously described [16]. HBE cells were grown on six-well culture plates in BEGM. Hydrocortisone was removed from the medium $24 \mathrm{~h}$ prior to stimulation and all stimulations were performed in this hydrocortisone-free medium.

\section{Virus}

WI-38 cells were purchased from the American Type Culture Collection (Manassas, VA). HRV-16 viral stocks were propagated in WI-38 cells and purified by centrifugation through sucrose to remove ribosomes and soluble factors of WI-38 origin as previously described [17]. 
HBE cells were infected with $10^{5.5} 50 \%$ tissue cultureinfective dose (TCID50) U/ml, which equates (approximately) to a multiplicity of infection of 1 .

\section{RNA extraction and real-time RT-qPCR}

Total cellular RNA was extracted from cultured epithelial cells with TRIzol $\left(1 \mathrm{ml}\right.$ per $\left.10 \mathrm{~cm}^{2}\right)$ as previously described [18]. Yield of RNA was quantified based upon absorbance at $260 \mathrm{~nm}$. RNA was DNase treated with DNase I (Ambion, Austin, TX) and then requantified. One $\mu \mathrm{g}$ of RNA was reverse transcribed with both Oligo $\mathrm{dT}$ and random hexamers with Superscript III, followed by PCR amplification in the presence of specific forward and reverse primers and fluorescently labeled probes for each gene of interest. Gene expression for CXCL10, CCL5, Viperin, IL-6, CXCL5, and HERC5 were quantified using the Applied Biosystems Model 7900 Sequence Detector (Foster City, CA). Analysis of the housekeeping gene GAPDH was performed on each sample using a primer and probe kit obtained from Applied Biosystems. Fold induction of each gene was calculated using the formula $2^{-\triangle \Delta C T}$ as previously described [19]. Primers and probes for CXCL10, CCL5 and viperin were as follows: CXCL10 forward primer 5'-GAAATTATTCCTGCAA GCCAATTT-3'; reverse primer 5'-TCACCCTTCT TTTTCATTGTAGCA-3'; probe 5'-FAM-TCCACGTG TTGAGATCA-MGB-3', CCL5 forward primer 5'TCTGCGCTCCTGCATCTG-3'; reverse primer 5'-AG TGGGCGGGCAATGTAG-3'; probe 5'-FAM-ATTCCT CGGACACCACACCCTGCTG-MGB-3', and viperin forward primer 5'-CCTGCTTGGTGCCTGAATCT-3'; reverse primer 5' - GCGCATATATTCATCCAGAATA AGG-3'; probe 5'-FAM-ACCAGAAGATGAAAGACTMGB-3'. Gene expression kits were purchased from Applied Biosystems for IL-6 (Hs00985639_m1), CXCL5 (Hs01099660_g1), and HERC5 (Hs00180943_m1).

\section{ELISAs}

CXCL10, CCL5 and CXCL5 protein levels were assayed by enzyme-linked immunosorbent assay using matched antibody pairs (R\&D Systems, Minneapolis, MN).

\section{siRNA knockdown of IRF7}

Subconfluent HBE cells were transfected with either 10 nM of a siRNA targeted to IRF7 or to a nontargeting siRNA, all-star control (Qiagen, Toronto, ON) for $24 \mathrm{~h}$ at $37^{\circ} \mathrm{C}$ using Lipofectamine RNAiMAX (Life Technologies) in BEGM without antibiotics. After transfection, medium was changed to BEGM without hydrocortisone and cells were allowed to recover for $24 \mathrm{~h}$. Cells were then infected with HRV-16, and supernatants, whole-cell lysates and total RNA were collected $24 \mathrm{~h}$ post infection. The specific forward siRNA sequences used were as follows: IRF7
siRNA \#1, 5'-CCCGAGCTGCACGTTCCTATA-3'; IRF7 siRNA \#2, 5' -CTGGAAGCACTTCGCGCGCAA-3'.

\section{Western blots}

After stimulation, cells were washed with HBSS and then scraped in lysis buffer (1\% Triton X-100, 1X mini complete, $1 \mathrm{mM}$ PMSF, $2 \mathrm{mM}$ sodium orthovanadate, $20 \mathrm{mM}$ sodium pyrophosphate, and $50 \mathrm{mM}$ sodium fluoride). Lysates were incubated on ice for $10 \mathrm{~min}$, frozen at $-80{ }^{\circ} \mathrm{C}$, thawed, sonicated, and centrifuged at $10,000 \times \mathrm{g}$ for $5 \mathrm{~min}$ at $4{ }^{\circ} \mathrm{C}$. Protein concentrations for the Triton X-100-soluble lysates were quantified using a DC Protein Assay (Bio-Rad, Montreal, Quebec, Canada). Equivalent amounts of protein were separated by SDSPAGE, and proteins were then transferred to a nitrocellulose membrane. Membranes were blocked with $5 \%$ skim milk for $1 \mathrm{~h}$ and probed with either $1 \mu \mathrm{g} / \mathrm{mL}$ of the IRF7 antibody or a 1:1000 dilution of the viperin antibody overnight at $4{ }^{\circ} \mathrm{C}$. Membranes were washed and then incubated for $1 \mathrm{~h}$ with a 1:10,000 dilution of horseradish peroxidase-conjugated anti-mouse Ig antibody (Jackson ImmunoResearch Laboratories, Inc., West Grove, PA). Proteins were visualized with a Pierce ECL substrate reagent (Thermo Scientific, Rockford, IL). Equal loading was further determined probing with an antibody against GAPDH.

\section{Gene expression profiling}

Total RNA samples were shipped on dry ice to Expression Analysis Inc. (Durham, NC). Purified RNA was quantified using a Nanodrop spectrophotometer and quality was determined using the Agilent (Santa Clara, CA) 2100 Lab-on-a-Chip System. The RNA samples were processed using the Enzo Single Round RNA Amplification and Biotin Labeling System (Enzo, New York, NY), and hybridized to Primeview microarrays (Affymetrix, Santa Clara CA). The raw microarray data is available at the Gene Expression Omnibus repository under accession GSE70190.

The microarray data was analyzed in $\mathrm{R}$ software for statistical computing (https://www.r-project.org/). The quality of the microarray data was assessed with ArrayQualityMetrics [20]. The data was preprocessed employing the RMA algorithm [21]. A custom chip description file (primeviewhsentrezg, version 19) was used to map probe sets to genes [22]. The data was filtered to differentiate between relevant gene expression signals and noise [23]. Differentially expressed genes were identified using Linear models for microarray data (LIMMA), with False Discovery Rate (FDR) control for multiple testing [24]. Pathways analysis was performed using InnateDb. Upstream regulators were identified using Ingenuity Systems software [25]. The IRF7 gene network was 
reconstructed using experimentally supported findings from the Ingenuity Systems Knowledgebase [14].

\section{Statistical analysis}

For assessments of mRNA and protein expression for individual genes of interest, normally distributed data were analyzed using one-way analysis of variance (ANOVA) with student Newman-Keuls post hoc analysis. Analysis of paired comparisons were performed using t-tests. Data that were not normally distributed were analyzed using Kruskal-Wallis ANOVA followed by Wilcoxon matched-pairs signed-rank test. Statistical hypotheses were two-sided and values of $p<0.05$ were considered significant.

\section{Results}

Rhinovirus-induced gene expression patterns in human bronchial epithelial (HBE) cells

Primary HBE cells from 5 donors were transfected with siRNA that targets IRF7 or a non-silencing siRNA control (all-star control). The HBE cells were allowed to recover for $24 \mathrm{~h}$, and then they were exposed to HRV or medium control for another $24 \mathrm{~h}$. As illustrated in Fig. 1, HRV induced the expression of IRF7 protein in HBE cells that were transfected with control reagents (all-star control siRNA, lipid reagent alone, medium only), but not in HBE cells that were transfected with siRNA reagents that target IRF7 (siRNA \#1, siRNA \#2). Of note, IRF7 protein was not induced by the all-star control siRNA in HBE cells in the absence of HRV exposure.

Next, global patterns of gene expression were profiled on microarrays. Initially, we analyzed the data pertaining to the response to HRV in HBE cells transfected with the all-star control. As illustrated in Fig. 2, 437 genes were upregulated in the response and 139 genes were

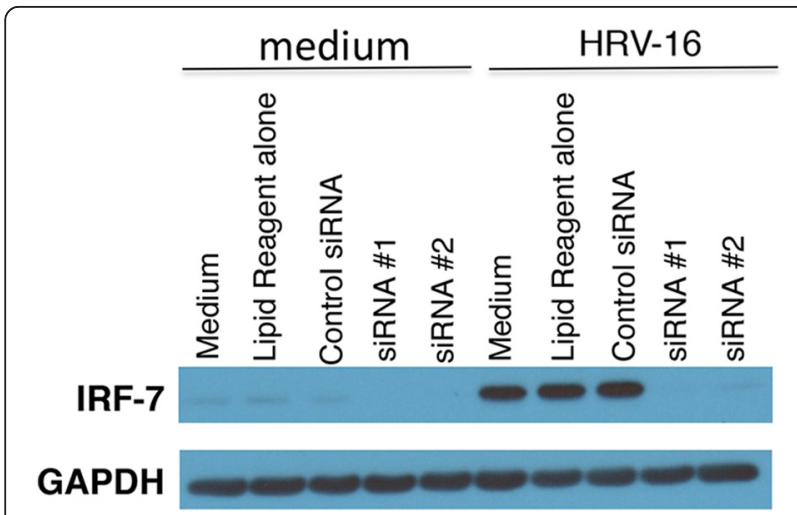

Fig. 1 Knockdown of IRF7 protein in HBE cells. HBE cells were transfected with the transfection reagent only (lipid reagent alone), the all-star control siRNA (control siRNA), or two independent siRNAs that target IRF7 (siRNA \#1, siRNA \#2). The cells were allowed to recover, and then exposed to HRV. Protein expression was measured by Western Blot. Data are representative of $n=4$

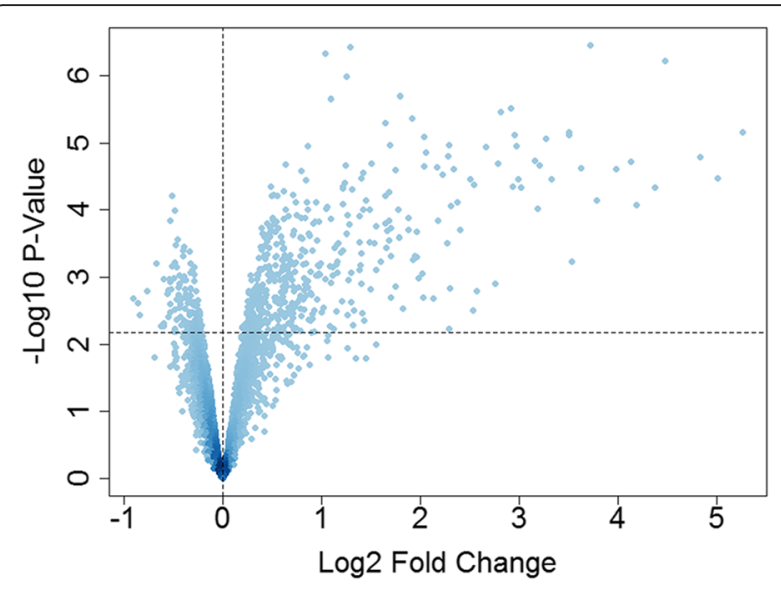

Fig. 2 Gene expression patterns in HRV-induced HBE cells. HBE cells from 5 donors were transfected with a non-silencing siRNA (allstar control), allowed to recover, and then cultured in the presence or absence of HRV for $24 \mathrm{~h}$. Gene expression was profiled on microarrays. The dashed horizontal line indicates FDR $<0.05$

downregulated (False Discovery Rate $(\mathrm{FDR})<0.05$, Additional file 1). The biological function of the differentially expressed genes was interrogated using InnateDb. The data showed the upregulated genes were mainly involved in type I and II interferon signaling, RIG-I/ MDA5 signaling, antigen processing and presentation, and apoptosis (Additional file 2). The downregulated genes were not strongly enriched for immune-related pathways, therefore we utilized the Gene Ontology (GO) database to interrogate their function. This analysis showed that the downregulated genes were enriched with the GO biological process term epithelial development (GO0060429; ALDH3A2, CTGF, DKK1, EMP1, ERRFI1, INSR, JAG1, KRT15, PTHLH, SCEL, SOX21; adjusted $p$-value $=0.0079$ ).

\section{IRF7 is a putative molecular driver of interferon-induced gene networks}

Upstream regulator analysis was employed to infer the molecular drivers that give rise to HRV-induced gene expression patterns in HBE cells (transfected with the all-star control) [25]. In this analysis, the differentially expressed genes from Fig. 2 were tested for enrichment of downstream target genes of known transcriptional regulators and other signal transduction pathways. As illustrated in Table 1, the most significant candidate drivers were IFNG, IFNA2, IRF7, TNF, and IFNL1. Notably, IRF7 was the only driver amongst these candidates that was also upregulated in the response (Additional file 1). To illustrate the role of IRF7 in the regulation of HRV responses, we utilized experimentally supported findings from the Ingenuity Systems database to reconstruct the IRF7 network [14]. The resulting network is shown in Fig. 3. 
Table 1 Identification of molecular drivers of gene expression patterns in HRV-induced HBE cells

\begin{tabular}{|c|c|c|c|}
\hline Upstream regulator & Predicted activation state & Activation Z-score & $P$-value of overlap \\
\hline IFNG & Activated & 10.890 & $1.08 \mathrm{E}-90$ \\
\hline IFNA2 & Activated & 8.106 & 9.79E-85 \\
\hline IRF7 & Activated & 8.284 & 1.76E-71 \\
\hline TNF & Activated & 9.828 & $3.92 \mathrm{E}-67$ \\
\hline IFNL1 & Activated & 6.936 & $1.02 \mathrm{E}-62$ \\
\hline IFNAR & Activated & 6.306 & 5.99E-55 \\
\hline STAT1 & Activated & 6.317 & $2.32 \mathrm{E}-54$ \\
\hline IFNB1 & Activated & 5.436 & $3.64 \mathrm{E}-50$ \\
\hline STAT3 & Activated & 4.185 & $9.25 E-48$ \\
\hline IRF1 & Activated & 5.780 & $6.24 \mathrm{E}-46$ \\
\hline NFkB (complex) & Activated & 6.850 & $1.41 \mathrm{E}-39$ \\
\hline TLR3 & Activated & 5.327 & $5.45 \mathrm{E}-39$ \\
\hline IL1B & Activated & 6.926 & 1.29E-37 \\
\hline CD40LG & Activated & 4.739 & $8.56 \mathrm{E}-35$ \\
\hline OSM & Activated & 5.463 & 1.99E-34 \\
\hline TLR4 & Activated & 5.165 & 3.64E-33 \\
\hline NKX2-3 & Inhibited & -7.703 & $1.50 \mathrm{E}-58$ \\
\hline MAPK1 & Inhibited & -6.867 & $5.29 E-58$ \\
\hline TRIM24 & Inhibited & -6.002 & 1.67E-44 \\
\hline IL1RN & Inhibited & -6.336 & $2.84 \mathrm{E}-35$ \\
\hline
\end{tabular}

Knockdown of IRF7 perturbs HBE responses to rhinovirus We next examined the impact of IRF7 knockdown on the HRV response. Gene expression patterns were compared between HRV-induced HBE cells transfected with IRF7-siRNA \#1 versus the all-star control. The data showed that knockdown of IRF7 resulted in decreased expression of 447 genes, and increased expression of 181 genes (Additional file 3, Fig. 4). The downregulated genes were mainly involved in type I and II interferon signaling, Toll-like receptor signaling, NOD-like receptor signaling, and RIG-I/MDA5 signaling (Additional file 4). As a supplementary analysis, we also examined the function of the downregulated genes after removal of known IRF7 target genes. These residual genes were associated with NOD-like receptor signaling pathways, trafficking of connexons, and metabolic pathways (Additional file 5). The upregulated genes were involved in the response to oxidative stress (GO:0006979; ADA, ARL6IP5, CYGB, FOS, MMP14, NET1, NQO1, PLK3, SNCA, TRPC6, UCP2; $p$-value $=0.0004$ ) and inflammation (GO:0050729; ADORA2B, C3, IL1RL1, IL33, SERPINE1, TNFSF11; $p$-value $=0.0005)$, although it is noteworthy that these findings were not significant after adjustment of the $p$-values for multiple testing.

We next wanted to investigate the part of the HRV response that was insensitive to IRF7 knockdown. In HBE cells that were transfected with the all-star control
siRNA, 576 genes were differentially expressed after HRV exposure, but only 177 of these genes (31\%) were sensitive to IRF7 knockdown (Fig. 5). Moreover, the IRF7 gene network depicted in Fig. 3 contained 86 genes, and only 41 of these (47\%) were sensitive to IRF7 knockdown (Fig. 4, Additional file 6). We analyzed these HRV responsive genes that were insensitive to IRF7 knockdown using upstream regulator analysis. The data showed the highest ranking candidate transcription factors driving this response were NF- $\mathrm{B}$, STAT1, STAT3, and IRF1 (Additional file 7).

\section{Validation of the microarray findings}

To confirm the findings from the microarray experiment, a subset of genes were selected for validation at the mRNA and/or protein levels. As illustrated in Fig. 6, Reverse Transcription quantitative PCR (RT-qPCR) analysis demonstrated that knockdown of IRF7 in HBE cells followed by exposure to HRV resulted in reduced expression of CCL5, CXCL10, HERC5, IL-6, RSAD2 (viperin), and increased expression of CXCL5, and this was consistent with the microarray data (Additional file 3). Expression levels of CCL5, CXCL5, CXCL10, and viperin were also examined at the protein level (Fig. 7). These data confirmed that the mRNA expression changes observed upon siRNA knockdown were associated with parallel changes at the protein level, although 


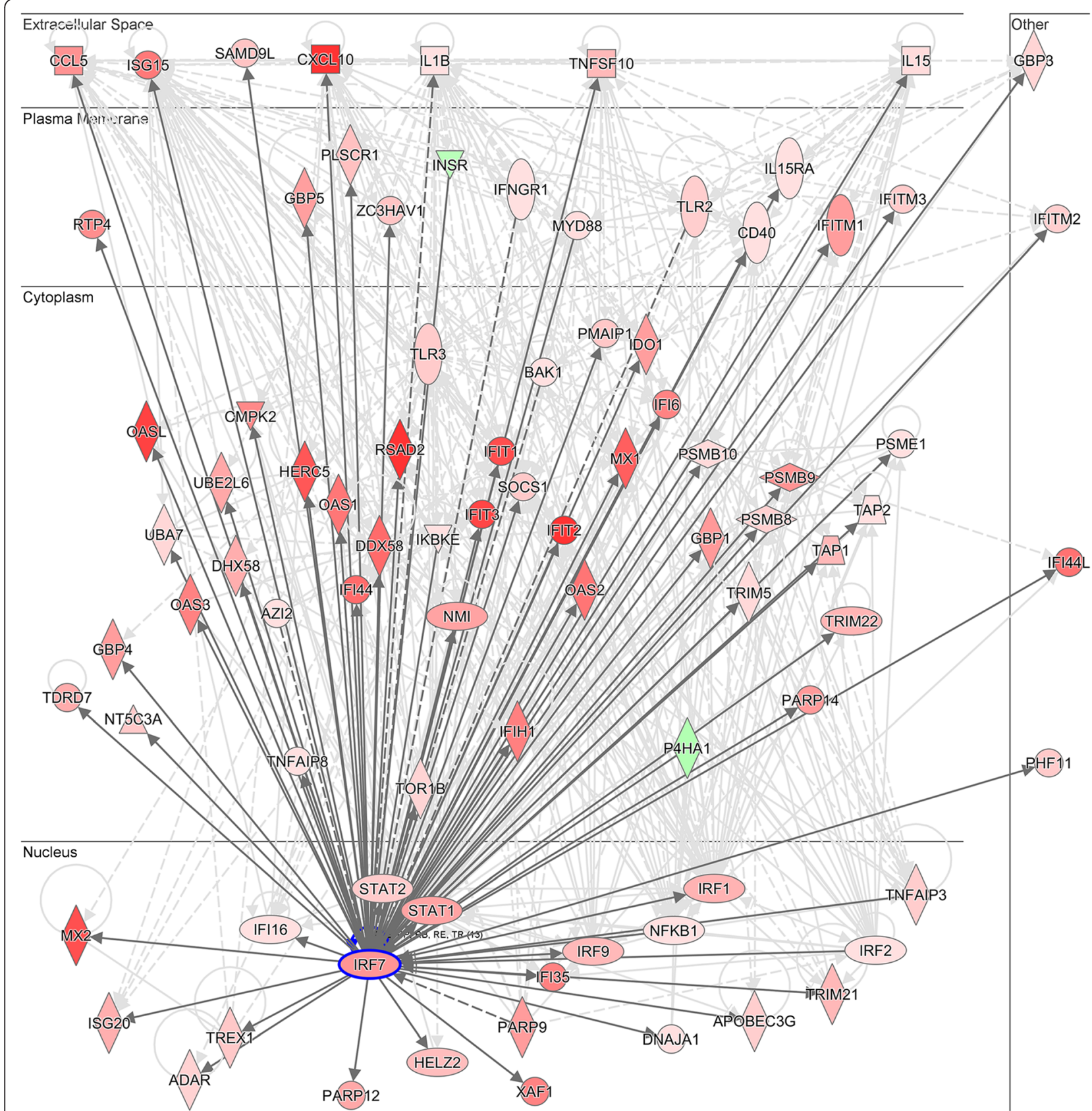

Fig. 3 IRF7 is a putative molecular driver of HBE responses to HRV. Differentially expressed genes were identified in HRV-induced HBE cells (Fig. 2), and then the wiring diagram of the IRF7 gene network was reconstructed from these data employing experimentally supported findings from published studies. Solid and dashed lines indicate direct and indirect interactions respectively. Genes colored red were upregulated in the response and genes colored green were downregulated

these changes were more pronounced with siRNA \#2 than with siRNA \#1.

\section{Discussion}

IRF7 is a master regulator of type I interferon gene expression and antiviral immunity, but the role of IRF7 in HBE responses to HRV has not been previously investigated. Employing microarray profiling we showed that hundreds of genes were upregulated in HBE upon HRV infection. Upstream regulator analysis [25] suggested that IRF7 was a major molecular driver of this response. We then showed that knockdown of IRF7 in HBE altered HRV responses, by decreasing the upregulation of genes involved in type I and II interferon signaling and innate immunity (toll-like receptor signaling, NOD-like receptor signaling, RIG-I/ 


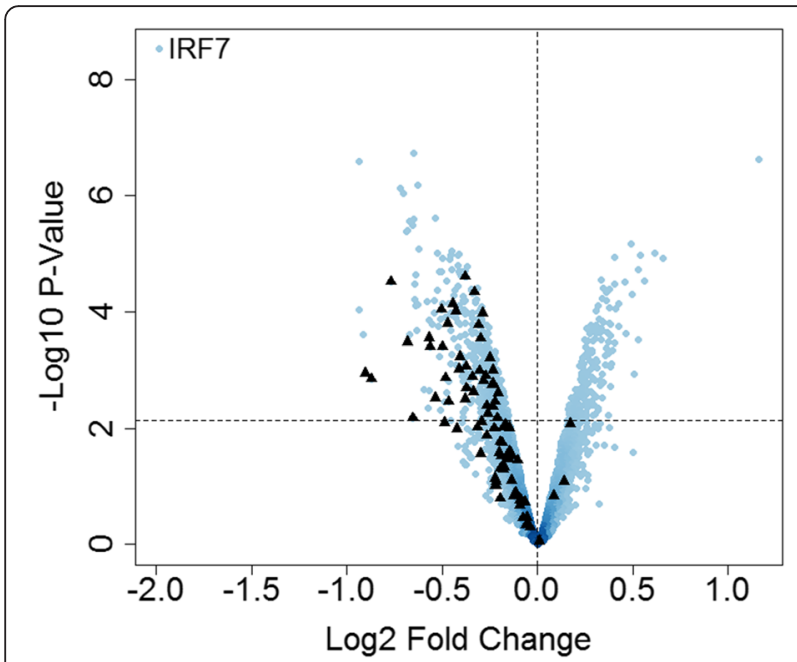

Fig. 4 Knockdown of IRF7 perturbs HBE cells responses to HRV. HBE cells from five donors were transfected with siRNA that targets IRF7 (siRNA \#1) or the non-silencing all-star control, allowed to recover, and then exposed to HRV for $24 \mathrm{~h}$. Gene expression was profiled on microarrays. The data was compared between HRV-induced cells treated with IRF7-siRNA \#1 versus the all-star control. Genes with negative values on the horizontal axis were decreased by IRF7 knockdown, whereas those with positive values were increased. The black triangles show the location of genes from the IRF7 network. The dashed horizontal line indicates FDR $<0.05$

MDA5 signaling), and increasing the expression of genes that promote inflammation (C3, CXCL5, IL-33, IL1RL1) and the response to oxidative stress. We validated a subset of the differentially regulated genes (CCL5, CXCL5, CXCL10, HERC5, IL-6, RSAD2/ viperin) at the mRNA and/or protein levels, thus confirming the findings from the microarray experiment. The data also showed that only around $30 \%$ of the HRV responsive genes and half of known IRF7 target

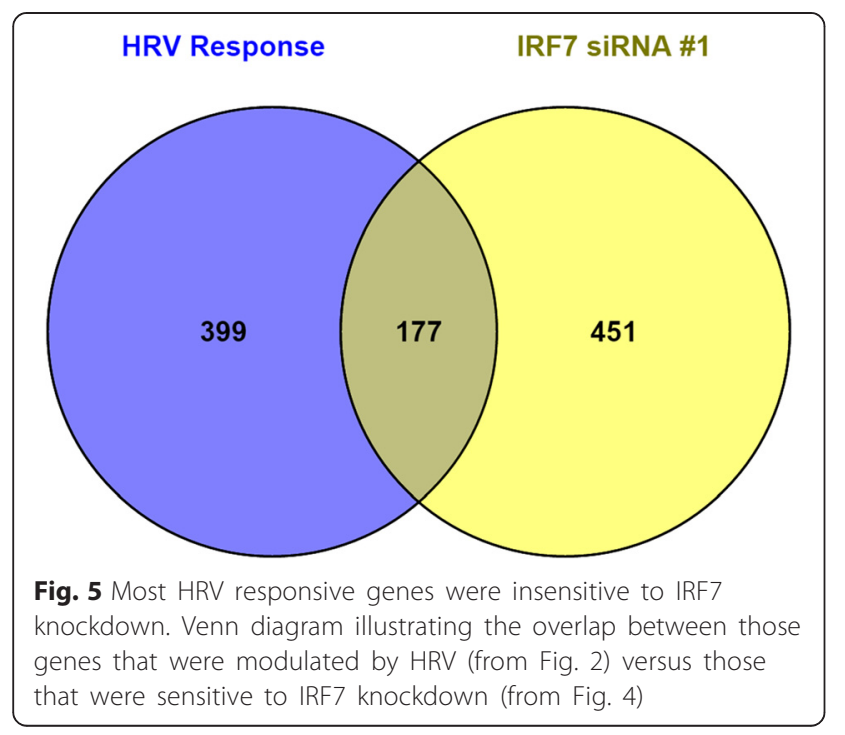

genes were sensitive to IRF7 knockdown, and these genes exhibited modest fold changes in our experiments (less than 2 fold), suggesting that other transcription factors are likely to be important in the regulation of interferon gene expression programs in HBE cells. Upstream regulator analysis of the part of the HRV response that was insensitive to IRF7 knockdown suggested roles for NF-kB, STAT1, STAT3, and IRF1 in the regulation of the response. Overall, our findings demonstrate that IRF7 regulates the expression of genes involved in antiviral immunity, inflammation, and the response to oxidative stress in HBE cells during HRV infections, and also suggest that additional transcription factors play a major role in the regulation of this response.

We showed that the upregulation of CCL5, CXCL10, HERC5, IL-6, and RSAD2 by HRV was reduced by IRF7 knockdown in HBE cells. Regulation of these genes by IRF7 has been previously reported in other contexts [26-29]. CCL5 (RANTES) is a potent chemoattractant for $\mathrm{T}$ cells, monocytes, and eosinophils. CCL5 was upregulated in nasal wash samples from wheezing, HRV-infected infants in comparison to their nonwheezing counterparts [30]. CXCL10 is chemotactic for $\mathrm{T}$ cells and natural killer cells, and was strongly upregulated in airway epithelial cells during HRV infections [31]. CXCL10 promotes airways inflammation and airways hyperresponsiveness in mouse models of asthma [32]. HERC5 is an E3 protein ligase that mediates ISGylation of protein targets. It catalyzes the conjugation of ISG15 onto IRF3, which inhibits the ubiquitination and degradation of IRF3 by preventing IRF3 from interacting with Pin1. This results in the sustained activation of IRF3 and the antiviral response [33]. IL-6 promotes the acute phase response, stimulates $\mathrm{T}$ cell responses and antibody production, and it is increased during symptomatic rhinovirus infections [34]. IRF7 was required for IL-6 production by poly (I-C) stimulated THP-1 monocytes [29]. In contrast, IRF7 was dispensable for IL-6 production in plasmacytoid and myeloid dendritic cells stimulated by TLR7 or TLR9 ligands [10]. Viperin (RSAD2) is an antiviral protein that can inhibit influenza budding from the plasma membrane by interfering with lipid rafts [35]. Knockdown of viperin in airway epithelial cells increased HRV replication [13]. It is not possible to determine from our experiments if IRF7 regulates HRV-induced airway inflammatory responses in vivo, however, Girkin et al. showed that intranasal delivery of siRNA that targets IRF7 reduced the infiltration of neutrophils and macrophages in the bronchoalveolar lavage fluid of nonallergic mice after HRV-1B infection [36]. Taken together, these studies suggest that IRF7 regulates HRV- 


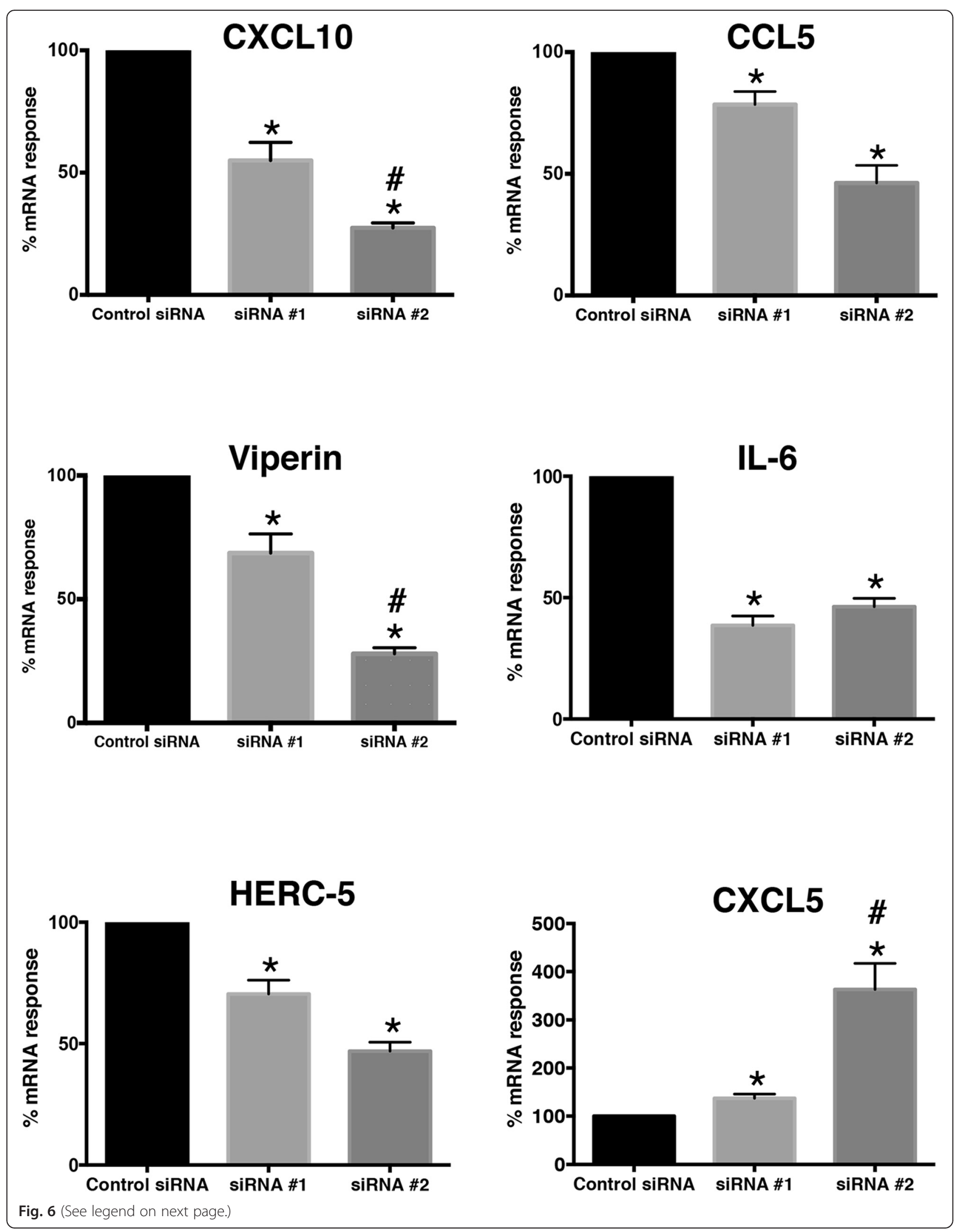


(See figure on previous page.)

Fig. 6 Validation of the microarray data at the mRNA level. HBE cells were transfected with the all-star control siRNA, or two independent siRNA reagents that target IRF7 (siRNA \#1, siRNA \#2). The cells were allowed to recover, and then exposed to HRV for 24 h. Gene expression levels were measured by real time RT-qPCR. The data for siRNA \#1 and siRNA \#2 are expressed as percent mRNA response relative to the all-star control. Data are mean \pm SEM from 4 experiments. Asterisks show significant differences compared to the all star control siRNA treatment. Hashmarks (\#) indicate significant differences between siRNA\#1 and siRNA\#2

induced gene network patterns and ensuing cellular immune responses in the airways.

In contrast to genes involved in antiviral defense, knockdown of IRF7 resulted in increased expression of CXCL5, IL-33, and IL1RL1. CXCL5 (ENA-78) is important for neutrophil homeostasis and recruitment to the lung during infections [37]. IL-33 is a nuclearassociated alarmin expressed in the airway epithelium. It signals through the ST2 receptor (IL1RL1), which is highly expressed on T-helper type 2 (Th2) lymphocytes and group 2 innate lymphoid cells (ILC2s) [38]. IL-33 is upregulated in the airways during HRVinduced asthma exacerbations [39]. Moreover, supernatants from HRV-infected epithelial cells stimulate the production of Th2 cytokines by Th2 and ILC2 cells in an IL-33 dependant manner [39]. IRF7 binds to the IL-33 promoter and regulates IL-33 transcription in human monocyte THP-1 cell lines [40]. Given that IL-33 is a potent driver of asthmatic ILC2 and Th2 responses, and we found that knockdown of IRF7 results in upregulation of IL-33, this suggests the hypothesis that promoting IRF7 responses during asthma exacerbations may have a dual beneficial effect of increasing antiviral immunity and supressing IL-33 responses.

Knockdown of IRF7 decreased expression of genes involved in Nod-like receptor signaling, and increased expression of genes involved in the response to oxidative stress (e.g. cytoglobin (CYGB), NAD(P)H:quinone acceptor oxidoreductase 1 (NQO1), alpha-synuclein (SNCA), short transient receptor potential channel 6 (TRPC6)) [41-44]. In this context it is noteworthy that the generation of reactive oxygen species during HRV infection compromises epithelial barrier function, and this can lead to secondary bacterial infections $[45,46]$. HRV infection stimulates the epithelial production of reactive oxygen species by triggering the dsRNA receptor Nod-like receptor X-1 (NLRX-1) [47]. Cross-talk between oxidative stress pathways and IRF7 signaling was reported in the context of respiratory syncytial virus infection [48]. The role of IRF7 in the regulation of Nodlike receptor signaling and oxidative stress pathways during HRV infections merits further investigation in follow-up studies.
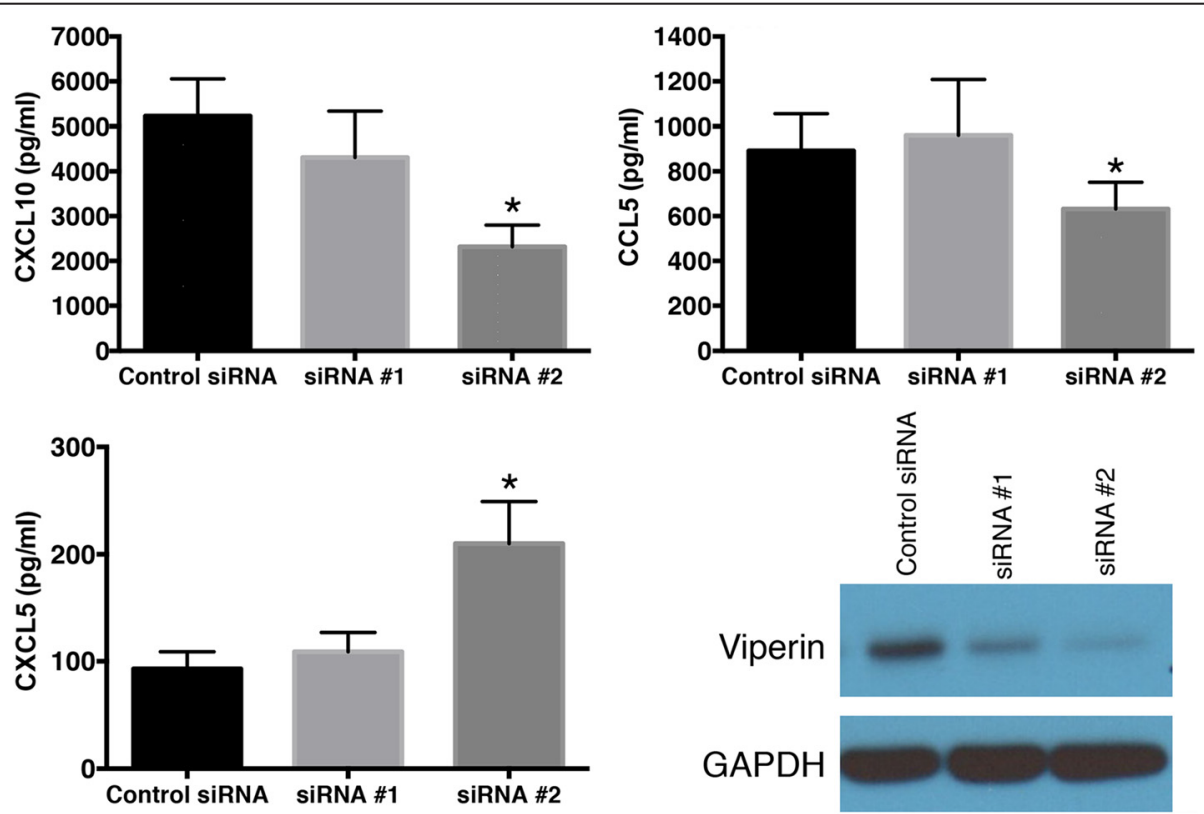

Fig. 7 Validation of the microarray data at the protein level. HBE cells were transfected with the all-star control siRNA, or two independent siRNA reagents that target IRF7 (siRNA \#1, siRNA \#2). The cells were allowed to recover, and then exposed to HRV for 24 h. Protein levels for CCL5, CXCL5, CXCL10 were measured by ELISA and are shown as mean \pm SEM from 4 experiments. Asterisks show significant differences compared to the all star control siRNA treatment. Expression of viperin protein was assessed by western blot. Data are representative of 4 such experiments 
Upstream regulator analysis demonstrated that IRF7 was the most significant candidate molecular driver that was upregulated in the HRV response. However, our siRNA studies showed that only about $30 \%$ of the HRV responsive genes and half of known IRF7 target genes were sensitive to IRF7 knockdown. Whilst variable and incomplete knockdown and the potential for nonspecific activation of antiviral pathways is a well-known limitation of siRNA technology, an alternative explanation of the data is that other transcription factors may be playing a role. Employing upstream regulator analysis we showed that NF-kB, STAT1, STAT3, and IRF1 were candidate drivers of the part of the HRV response that was insensitive to IRF7 knockdown. In this context it is noteworthy we have reported that HRV-induced upregulation of CXCL10 in bronchial epithelial cells was dependent on IRF1 [49]. Wang et al. have also reported that knockdown of IRF3 abrogated HRV-induced type I and III interferon responses in BEAS-2B epithelial cells [5]. Taken together, these observations suggest that interferon responses in HRV-induced HBE cells can be variably mediated by interactions between IRF1, IRF3, and IRF7.

This study has limitations that should be acknowledged. First, the gene expression studies were conducted at a single time point post infection $(24 \mathrm{~h})$, so it was not possible to determine the role of IRF7 in the kinetics of the response. Second, siRNA-mediated gene silencing was used to investigate the role of IRF7 in the regulation of gene expression patterns, and from this data it was not possible to determine if IRF7 was playing a direct or indirect regulatory role. Third, prior knowledge was used to infer the molecular drivers of the response, and this analysis cannot reveal novel molecular drivers. This latter limitation can be addressed using unbiased datadriven algorithms to infer the molecular drivers of the response [50]. Although we acknowledge that the use of epithelial cells grown in submersion culture, as opposed to differentiated air-liquid interface cultures, may be considered a limitation of the current study, we believe that the data obtained are still highly relevant. This is based on the observation that the profile of HRVupregulated genes in these cultures, in both the present and in an earlier study [51], aligns closely with those observed in nasal epithelial scrapings from individuals experimentally infected with HRV in vivo [13].

\section{Conclusion}

Our study demonstrates that IRF7 regulates the expression of antiviral, inflammatory and oxidative stress response pathways during HRV infections in HBE cells, and suggests that additional transcription factors play an important role in the response.

\section{Availability of supporting data}

The microarray data are available at the Gene Expression Omnibus repository (accession GSE70190).

\section{Additional files}

\begin{abstract}
Additional file 1: This excel file contains the list of differentially expressed genes that were induced by HRV in HBE cells transfected with the all-star control siRNA. (XLSX $71 \mathrm{~kb})$
\end{abstract}

Additional file 2: This excel file contains the list of biological pathways that were upregulated by HRV in HBE cells transfected with the all-star control siRNA. (XLSX $23 \mathrm{~kb}$ )

Additional file 3: This excel file contains the list of differentially expressed genes identified in HRV-induced HBE cells transfected with IRF7-siRNA \#1 versus the all-star control siRNA. (XLSX $77 \mathrm{~kb}$ )

Additional file 4: This excel file contains the list of biological pathways that were decreased in HRV-induced HBE cells transfected with IRF7-siRNA\#1 versus the all-star control siRNA. (XLSX $13 \mathrm{~kb}$ )

Additional file 5: This excel file contains the results of a pathways analysis of remaining genes (after removal of known IRF7 target genes) that were decreased in HRV-induced HBE cells transfected with IRF7-siRNA\#1 versus the all-star control siRNA. (XLSX 11 kb)

Additional file 6: This excel file contains the results of a differential gene expression analysis of IRF7 gene networks in HBE cells transfected with IRF7-siRNA\#1 versus the all-star control siRNA. (XLSX $18 \mathrm{~kb}$ )

Additional file 7: This excel file contains the results from an Upstream regulator analysis of the HRV responsive genes in $\mathrm{HBE}$ cells that were insensitive to IRF7 knockdown. (XLSX 11 kb)

\section{Abbreviations}

ADA: adenosine deaminase; ADORA2B: adenosine A2b receptor; ALDH3A2: aldehyde dehydrogenase 3 family member A2; ANOVA: analysis of variance; ARL6IP5: ADP-ribosylation factor-like 6 interacting protein 5; BEGM: bronchial epithelial cell growth medium; C3: complement component 3; CCL5: chemokine (C-C motif) ligand 5, also known as regulated on activation, normal T cell expressed and secreted (RANTES); CDHR3: Cadherinrelated family member 3; CTGF: connective tissue growth factor;

CXCL10: chemokine (C-X-C motif) ligand 10; CXCL5: chemokine (C-X-C motif) ligand 5; CYGB: cytoglobin; DKK1: dickkopf WNT signaling pathway inhibitor 1; ELISA: enzyme-linked immunosorbent assay; EMP1: epithelial membrane protein 1; ERRFI1: ERBB receptor feedback inhibitor 1; FBS: fetal bovine serum; FDR: False Discovery Rate; FOS: FBJ murine osteosarcoma viral oncogene homolog; GAPDH: glyceraldehyde-3-phosphate dehydrogenase; GO: Gene Ontology; HBE: human bronchial epithelial cells; HBSS: Hank's balanced salt solution; HERC5: HECT and RLD domain containing E3 ubiquitin protein ligase 5; HRV: human rhinovirus; HRV-16: human rhinovirus type 16; HRV-C: human rhinovirus type C; IFNG: interferon gamma; IFNL1: interferon, lambda 1; IL-1RL1: interleukin 1 receptor-like 1; IL33: interleukin-33; ILC2: group 2 innate lymphoid cells; INSR: insulin receptor; IRF7: interferon regulatory factor 7; ISG15: interferon stimulated gene that encodes interferon-induced 17 kDa protein; JAG1: jagged 1, KRT15, keratin 15 type l; LIMMA: linear models for microarray data; MDA5: melanoma differentiation associated gene 5; MMP14: matrix metallopeptidase 14; MX1: MX dynamin-like GTPase 1; NET1: neuroepithelial cell transforming 1; NF-kB: nuclear factor kappa-light-chain-enhancer of activated B cells; NODlike: receptor nucleotide-binding oligomerization domain receptor; NQO1: NAD(P)H dehydrogenase, quinone 1; OAS: 2'-5'-oligoadenylate synthetase 1; PKR: protein kinase RNA-activated; PLK3: polo-like kinase 3; PRR: pathogen recognition receptor; PTHLH: parathyroid hormone-like hormone; RANTES: regulated on activation, normal T cell expressed and secreted; RIG-I: retinoic acid-inducible gene 1; RT-qPCR: reverse transcription quantitative polymerase chain reaction; SCEL: sciellin; SDS-PAGE: sodium dodecyl sulfate polyacrylamide gel electrophoresis; SERPINE1: serpin peptidase inhibitor clade E; siRNA: small interfering RNA; SNCA: synuclein, alpha; SOX21: sex determining region Y box 21; Th2: T-helper type 2; TLR: toll-like receptor; TNF: tumor necrosis factor; TNFSF11: tumor necrosis 
factor (ligand) superfamily member 11, also known as receptor activator of nuclear factor-KB ligand (RANKL); TRPC6: transient receptor potential cation channel, subfamily C, member 6; UCP2: uncoupling protein 2.

\section{Competing interests}

The authors declare that they have no competing interests.

\section{Authors' contributions}

AB participated in the design and conception of the study, analyzed the gene expression profiling experiments and drafted the manuscript. SW carried out experiments, analyzed data, and helped to draft the manuscript DP conceived and designed the study, analyzed data, and helped to draft the manuscript. All authors read and approved the final manuscript.

\section{Acknowledgements}

This work was supported by the Department of Health, Government of Western Australia, and grant number 43923 from the Canadian Institutes of Health Research. AB is supported by a BrightSpark Foundation McCusker Fellowship. D. Proud holds a Canada Research Chair in Inflammatory Airway Disease.

\section{Author details}

${ }^{1}$ Telethon Kids Institute, University of Western Australia, PO Box 855, West Perth, WA 6872, Australia. ${ }^{2}$ Airway Inflammation Research Group, Snyder Institute for Chronic Diseases, and the Department of Physiology \& Pharmacology, University of Calgary Faculty of Medicine, Calgary, AB, Canada.

\section{Received: 20 October 2015 Accepted: 18 January 2016}

\section{Published online: 25 January 2016}

\section{References}

1. Leigh R, Proud D. Virus-induced modulation of lower airway diseases: pathogenesis and pharmacologic approaches to treatment. Pharmacol Ther. 2015;148:185-98.

2. Gern JE. The ABCs of rhinoviruses, wheezing, and asthma. J Virol. 2010; 84(15):7418-26.

3. Bochkov YA, Watters K, Ashraf S, Griggs TF, Devries MK, Jackson DJ, et al. Cadherin-related family member 3 , a childhood asthma susceptibility gene product, mediates rhinovirus C binding and replication. Proc Natl Acad Sci U S A. 2015.

4. Wang Q, Miller DJ, Bowman ER, Nagarkar DR, Schneider D, Zhao Y, et al. MDA5 and TLR3 initiate pro-inflammatory signaling pathways leading to rhinovirus-induced airways inflammation and hyperresponsiveness. PLoS Pathog. 2011;7(5), e1002070.

5. Wang Q, Nagarkar DR, Bowman ER, Schneider D, Gosangi B, Lei J, et al. Role of double-stranded RNA pattern recognition receptors in rhinovirus-induced airway epithelial cell responses. J Immunol. 2009:183(11):6989-97.

6. Slater L, Bartlett NW, Haas JJ, Zhu J, Message SD, Walton RP, et al. Coordinated role of TLR3, RIG-I and MDA5 in the innate response to rhinovirus in bronchial epithelium. PLoS Pathog. 2010;6(11), e1001178.

7. Ning S, Pagano JS, Barber GN. IRF7: activation, regulation, modification and function. Genes Immun. 2011;12(6):399-414.

8. Marie I, Durbin JE, Levy DE. Differential viral induction of distinct interferonalpha genes by positive feedback through interferon regulatory factor-7. EMBO J. 1998;17(22):6660-9.

9. Schoggins JW, Rice CM. Interferon-stimulated genes and their antiviral effector functions. Curr Opin Virol. 2011;1(6):519-25.

10. Honda K, Yanai $H$, Negishi $H$, Asagiri M, Sato M, Mizutani T, et al. IRF-7 is the master regulator of type-I interferon-dependent immune responses. Nature. 2005:434(7034):772-7.

11. Ciancanelli MJ, Huang SX, Luthra P, Garner H, Itan Y, Volpi S, et al. Infectious disease. Life-threatening influenza and impaired interferon amplification in human IRF7 deficiency. Science. 2015:348(6233):448-53.

12. Daffis S, Suthar MS, Szretter KJ, Gale Jr M, Diamond MS. Induction of IFNbeta and the innate antiviral response in myeloid cells occurs through an IPS-1-dependent signal that does not require IRF-3 and IRF-7. PLoS Pathog. 2009;5(10), e1000607.

13. Proud D, Turner RB, Winther B, Wiehler S, Tiesman JP, Reichling TD, et al. Gene expression profiles during in vivo human rhinovirus infection: insights into the host response. Am J Respir Crit Care Med. 2008;178(9):962-8.
14. Bosco A, Ehteshami S, Panyala S, Martinez FD. Interferon regulatory factor 7 is a major hub connecting interferon-mediated responses in virus-induced asthma exacerbations in vivo. J Allergy Clin Immunol. 2012;129(1):88-94.

15. Lewis TC, Henderson TA, Carpenter AR, Ramirez IA, McHenry CL, Goldsmith AM, et al. Nasal cytokine responses to natural colds in asthmatic children. Clin Exp Allergy. 2012;42(12):1734-44.

16. Churchill L, Chilton FH, Resau JH, Bascom R, Hubbard WC, Proud D. Cyclooxygenase metabolism of endogenous arachidonic acid by cultured human tracheal epithelial cells. Am Rev Respir Dis. 1989;140:449-59.

17. Sanders SP, Siekierski ES, Porter JD, Richards SM, Proud D. Nitric oxide inhibits rhinovirus-induced cytokine production and viral replication in a human respiratory epithelial cell line. J Virol. 1998:72:934-42.

18. Chomczynski P, Sacchi N. Single-step method of RNA isolation by acid guanidinium thiocyanate-phenol-chloroform extraction. Anal Biochem. 1987; 162:156-9.

19. Sanders SP, Siekierski ES, Richards SM, Porter JD, Imani F, Proud D. Rhinovirus infection induces expression of type 2 nitric oxide synthase in human respiratory epithelial cells in vitro and in vivo. J Allergy Clin Immunol. 2001;107:235-43.

20. Kauffmann A, Gentleman R, Huber W. arrayQualityMetrics-a bioconductor package for quality assessment of microarray data. Bioinformatics. 2009; 25(3):415-6.

21. Irizarry RA, Hobbs B, Collin F, Beazer-Barclay YD, Antonellis KJ, Scherf U, et al. Exploration, normalization, and summaries of high density oligonucleotide array probe level data. Biostatistics. 2003;4(2):249-64.

22. Dai M, Wang P, Boyd AD, Kostov G, Athey B, Jones EG, et al. Evolving gene/ transcript definitions significantly alter the interpretation of GeneChip data. Nucleic Acids Res. 2005;33(20), e175.

23. Lu J, Kerns RT, Peddada SD, Bushel PR. Principal component analysis-based filtering improves detection for Affymetrix gene expression arrays. Nucleic Acids Res. 2011:39(13), e86.

24. Smyth GK. Linear models and empirical bayes methods for assessing differential expression in microarray experiments. Stat Appl Genet Mol Biol. 2004;3:Article3.

25. Kramer A, Green J, Pollard Jr J, Tugendreich S. Causal analysis approaches in Ingenuity Pathway Analysis. Bioinformatics. 2014;30(4):523-30.

26. Lin R, Genin P, Mamane $Y$, Hiscott J. Selective DNA binding and association with the CREB binding protein coactivator contribute to differential activation of alpha/beta interferon genes by interferon regulatory factors 3 and 7. Mol Cell Biol. 2000;20(17):6342-53.

27. Goubau D, Romieu-Mourez R, Solis M, Hernandez E, Mesplede T, Lin R, et al. Transcriptional re-programming of primary macrophages reveals distinct apoptotic and anti-tumoral functions of IRF-3 and IRF-7. Eur J Immunol. 2009:39(2):527-40.

28. Barnes BJ, Richards J, Mancl M, Hanash S, Beretta L, Pitha PM. Global and distinct targets of IRF-5 and IRF-7 during innate response to viral infection. J Biol Chem. 2004;279(43):45194-207

29. Sweeney SE. Targeting interferon regulatory factors to inhibit activation of the type I IFN response: implications for treatment of autoimmune disorders. Cell Immunol. 2011;271(2):342-9.

30. Pacifico L, lacobini M, Viola F, Werner B, Mancuso G, Chiesa C. Chemokine concentrations in nasal washings of infants with rhinovirus illnesses. Clin Infect Dis. 2000;31(3):834-8.

31. Spurrell JC, Wiehler S, Zaheer RS, Sanders SP, Proud D. Human airway epithelial cells produce IP-10 (CXCL10) in vitro and in vivo upon rhinovirus infection. Am J Physiol Lung Cell Mol Physiol. 2005;289(1):L85-95.

32. Medoff BD, Sauty A, Tager AM, Maclean JA, Smith RN, Mathew A, et al. IFNgamma-inducible protein 10 (CXCL10) contributes to airway hyperreactivity and airway inflammation in a mouse model of asthma. J Immunol. 2002; 168(10):5278-86

33. Shi HX, Yang K, Liu X, Liu XY, Wei B, Shan YF, et al. Positive regulation of interferon regulatory factor 3 activation by Herc5 via ISG15 modification. Mol Cell Biol. 2010;30(10):2424-36.

34. Zhu Z, Tang W, Ray A, Wu Y, Einarsson O, Landry ML, et al. Rhinovirus stimulation of interleukin-6 in vivo and in vitro. Evidence for nuclear factor kappa B-dependent transcriptional activation. J Clin Invest. 1996; 97(2):421-30.

35. Wang $X$, Hinson ER, Cresswell $P$. The interferon-inducible protein viperin inhibits influenza virus release by perturbing lipid rafts. Cell Host Microbe. 2007;2(2):96-105. 
36. Girkin J, Hatchwell L, Foster P, Johnston SL, Bartlett N, Collison A, et al. CCL7 and IRF-7 mediate hallmark inflammatory and IFN responses following rhinovirus 1B infection. J Immunol. 2015;194(10):4924-30.

37. Mei J, Liu Y, Dai N, Hoffmann C, Hudock KM, Zhang P, et al. Cxcr2 and Cxcl5 regulate the IL-17/G-CSF axis and neutrophil homeostasis in mice. J Clin Invest. 2012;122(3):974-86.

38. Molofsky AB, Savage AK, Locksley RM. Interleukin-33 in tissue homeostasis, injury, and inflammation. Immunity. 2015;42(6):1005-19.

39. Jackson DJ, Makrinioti H, Rana BM, Shamji BW, Trujillo-Torralbo MB, Footitt J, et al. IL-33-dependent type 2 inflammation during rhinovirus-induced asthma exacerbations in vivo. Am J Respir Crit Care Med. 2014;190(12):1373-82.

40. Sun L, Zhu Z, Cheng N, Yan Q, Ye RD. Serum amyloid A induces interleukin33 expression through an IRF7-dependent pathway. Eur J Immunol. 2014; 44(7):2153-64.

41. Dinkova-Kostova AT, Talalay P. NAD(P)H:quinone acceptor oxidoreductase 1 (NQO1), a multifunctional antioxidant enzyme and exceptionally versatile cytoprotector. Arch Biochem Biophys. 2010;501(1):116-23.

42. le Thuy $\Pi$, Matsumoto $Y$, Thuy $T$, Hai H, Suoh M, Urahara Y, et al. Cytoglobin deficiency promotes liver cancer development from hepatosteatosis through activation of the oxidative stress pathway. Am Pathol. 2015;185(4):1045-60.

43. Hashimoto M, Hsu LJ, Rockenstein E, Takenouchi T, Mallory M, Masliah E. alpha-Synuclein protects against oxidative stress via inactivation of the cJun N-terminal kinase stress-signaling pathway in neuronal cells. J Biol Chem. 2002;277(13):11465-72.

44. Ding Y, Winters A, Ding M, Graham S, Akopova I, Muallem S, et al. Reactive oxygen species-mediated TRPC6 protein activation in vascular myocytes, a mechanism for vasoconstrictor-regulated vascular tone. J Biol Chem. 2011; 286(36):31799-809.

45. Comstock AT, Ganesan S, Chattoraj A, Faris AN, Margolis BL, Hershenson MB, et al. Rhinovirus-induced barrier dysfunction in polarized airway epithelial cells is mediated by NADPH oxidase 1. J Virol. 2011;85(13):6795-808.

46. Sajjan U, Wang Q, Zhao Y, Gruenert DC, Hershenson MB. Rhinovirus disrupts the barrier function of polarized airway epithelial cells. Am J Respir Crit Care Med. 2008;178(12):1271-81.

47. Unger BL, Ganesan S, Comstock AT, Faris AN, Hershenson MB, Sajjan US. Nod-like receptor $X-1$ is required for rhinovirus-induced barrier dysfunction in airway epithelial cells. J Virol. 2014;88(7):3705-18.

48. Fang L, Choudhary S, Tian B, Boldogh I, Yang C, Ivanciuc T, et al. Ataxia telangiectasia mutated kinase mediates NF-kappaB serine 276 phosphorylation and interferon expression via the IRF7-RIG-I amplification loop in paramyxovirus infection. J Virol. 2015;89(5):2628-42.

49. Zaheer RS, Proud D. Human rhinovirus-induced epithelial production of CXCL10 is dependent upon IFN regulatory factor-1. Am J Respir Cell Mo Biol. 2010;43(4):413-21.

50. Huynh-Thu VA, Irrthum A, Wehenkel L, Geurts P. Inferring regulatory networks from expression data using tree-based methods. PLoS One. 2010;5(9):e12776.

51. Proud D, Hudy MH, Wiehler S, Zaheer RS, Amin MA, Pelikan JB, et al. Cigarette smoke modulates expression of human rhinovirus-induced airway epithelial host defense genes. PLoS One. 2012;7, e40762.

\section{Submit your next manuscript to BioMed Central and we will help you at every step:}

- We accept pre-submission inquiries

- Our selector tool helps you to find the most relevant journal

- We provide round the clock customer support

- Convenient online submission

- Thorough peer review

- Inclusion in PubMed and all major indexing services

- Maximum visibility for your research

Submit your manuscript at www.biomedcentral.com/submit 